\title{
Philonsorbonne
}

15 | 2021

Année 2020-2021

\section{Lumière au fond du puits? Deschamps et le problème de la vérité}

Pierre BROSSARD

\section{OpenEdition}

1 Journals

Édition électronique

URL : https://journals.openedition.org/philonsorbonne/1860

DOI : 10.4000/philonsorbonne.1860

ISSN : $2270-7336$

Éditeur

Publications de la Sorbonne

Édition imprimée

Date de publication : 1 janvier 2021

Pagination : 153-167

ISSN : 1255-183X

\section{Référence électronique}

Pierre BROSSARD, «Lumière au fond du puits ? Deschamps et le problème de la vérité »,

Philonsorbonne [En ligne], 15 | 2021, mis en ligne le 03 février 2021, consulté le 10 juin 2021. URL:

http://journals.openedition.org/philonsorbonne/1860; DOI : https://doi.org/10.4000/philonsorbonne. 1860

(c) Tous droits réservés 


\title{
Lumière au fond du puits ? Deschamps et le problème de la vérité
}

\author{
Pierre BROSSARD
}

Qui peut savoir? Tout le monde, répondra-t-on ; c'est que, «si tous n'ont pas dans le fait également d'esprit, tous du moins en ont également en puissance, c'est-à-dire aptitude à en avoir ${ }^{1}$. De l'inégalité réelle des connaissances, on se gardera bien alors de conclure à l'inégalité naturelle du pouvoir de connaitre. Mais on pourrait aussi bien répondre : personne. Car le Philosophe voit que la plupart des hommes n'a guère cure des questions qu'il se pose - qu'en est-il de Dieu, de l'être, de la matière, de l'esprit ? Alors, se dit-il à lui-même, "voyant même qu'ils se moquaient souvent de ce que je voulais le savoir, j'ai soupçonné qu'il n'était point nécessaire que nous le sussions $»^{2}$; et pour échapper au ridicule, il acceptera de s'inclure, lui aussi, dans l'ignorance générale. Sans doute y a-t-il lieu, bien sûr, de distinguer entre les matières. D'une part, les sciences et les arts, utiles à tous, et donc intéressants pour chacun - et on affirmera alors l'égale dignité des esprits à s'en emparer. D'autre part, les spéculations métaphysiques, absconses et insolubles : ne pas pouvoir savoir, c'est de ce côté-là refuser de s'encombrer d'un inutile fardeau.

Toutefois, la difficulté demeure: il ne s'agit pas simplement de circonscrire les limites du savoir, ni d'établir en droit la capacité de tous à y prendre part, mais de se demander ce qu'il en est de sa circulation

1. Helvétius, De l'homme, section II, chap. XV, « De l'esprit», in Euvres complètes, Paris, Honoré Champion, 2011-2020, t. II, p. 152.

2. Voltaire, Le philosophe ignorant, IV, in Euvres complètes, Oxford, Voltaire Foundation, 1987, vol. 62, p. 34. 
effective dans le corps social. Car s'il y a un idéal des Lumières, c'est celui d'une diffusion, comme l'atteste la métaphore spatiale par laquelle elles se décrivent elles-mêmes : " nos travaux doivent avoir pour but, ou d'étendre les limites des places éclairées, ou de multiplier sur le terrain les centres de lumières ${ }^{3}{ }^{3}$, affirme Diderot. C'est d'abord l'extension des connaissances humaines qui est ainsi décrite : les deux voies de l'expérimentation et de la systématisation, esquissées dans les Pensées sur l'interprétation de la nature (1754), doivent collaborer à étendre le domaine réel du savoir. Mais c'est en même temps leur propagation d'un individu à l'autre -ainsi Diderot écrit-il à Necker, qui se targue de l'inutilité des lettres au regard de la science économique :

L'opinion, ce mobile dont vous connaissez toute la force pour le bien et pour le mal, n'est à son origine que l'effet d'un petit nombre d'hommes qui parlent après avoir pensé, et qui forment sans cesse, en différents points de la société, des centres d'instructions d'où les erreurs et les vérités raisonnées gagnent de proche en proche, jusqu'aux derniers confins de la cité, où elles s'établissent comme des articles de foi ${ }^{4}$.

L'affirmation du rôle de «l'homme de lettres »- qui sera aussi bien «littérateur» ou «philosophe» - est en même temps l'invention d'un espace public dans lequel doivent circuler «les erreurs et les vérités raisonnées ». Mais l'on se gardera bien de conclure, de la nécessité de cette circulation, à l'homogénéité du milieu où elle circule: sous le régime général de l'opinion s'opère en fait une déperdition, de sorte que ce qui était $s u$ par les "centres d'instructions » que sont les philosophes ne sera que cru par le peuple, chez qui tout devient «article de foi ». C'est que parmi ce dernier la raison est rare : « il n'a malheureusement pas le temps de la cultiver, de l'étendre et de s'en servir $\iota^{5}$. Les philosophes peuvent alors eux-mêmes définir leur position sociale à travers une image apocalyptique : " nous sommes ce petit nombre de têtes qui, placées sur le cou du grand animal, traînent après elles la multitude aveugle de ses queues $»^{6}$.

Les Lumières doivent donc prendre au sérieux l'obstacle que constitue, $\mathrm{du}$ point de vue du savoir, la structure inégalitaire du monde social: la propagation des rayons lumineux a affaire, ici comme ailleurs, à des phénomènes de réfraction qui en dévient la trajectoire. On se trouve alors au carrefour d'une double difficulté.

D'abord, celle de l'inégalité des progrès: précisément parce que le développement des sciences n'est pas simultanément celui de tous les esprits, on peut se demander jusqu'à quel point les progrès des connaissances

3. Diderot, Pensées sur l'interprétation de la nature, in Euvres, Paris, Robert Laffont, 19941997, vol. 1, p. 566.

4. Diderot, Lettre à Necker, 12 juin 1775, in Euvres, éd. cit., vol. 5, p. 1262.

5. Op. cit., p. 1263.

6. Ibid. 
humaines signifient en même temps l'amélioration générale de la société dans laquelle ils se produisent. On pourra alors faire valoir l'utilité présente de la raison, qui améliore en acte la condition de chacun ${ }^{7}$, ou au contraire insister sur l'hétérogénéité des progrès, qui dissimule trop mal l'inégalité des conditions : «les Sciences, les Lettres et les Arts étendent des guirlandes de fleurs sur des chaînes de fer $»^{8}$.

Ensuite, celle de l'usage social de la croyance: à supposer que tous les esprits puissent être éclairés, qu'en serait-il de l'unité du corps social ? Car les hommes semblent avoir besoin, pour ne pas s'entre-déchirer, d'une opinion commune qui leur interdit de le faire : qui jouera mieux ce rôle que la foi ? On retrouve ainsi le spectre de l'anarchie d'un monde sans Dieu, que Bayle avait déjà tenté d'écarter en affirmant qu'une société d'athées était possible. « Ôtez aux hommes l'idée d'un Dieu rémunérateur et vengeur, Silla et Marius se baignent alors avec délice dans le sang de leurs concitoyens $»{ }^{9}$, affirme l'adepte de la religion naturelle - qui bien sûr ne cherche nullement par là à soumettre les hommes à la tutelle théologique, mais seulement à « ce que la raison, de concert avec l'intérêt du genre humain, nous ordonne de croire $\gg{ }^{10}$. Mais n'est-on pas en droit alors de soupçonner qu'ici, la raison est moins contraignante que contrainte, et que la religion, même épurée en théisme, est encore une religion ${ }^{11}$ ? Et s'il est nécessaire que le peuple ne soit pas trop éclairé, ne sera-t-il pas symétriquement plus prudent de cantonner la liberté de spéculer à l'élite ? Ainsi Cléobule, qui incarne la figure d'un libertin retiré du monde et rompu à garder ses idées sous le manteau, douche-t-il les ambitions d'éducateur du jeune Ariste : "présenter la vérité à de certaines gens, c'est (...) introduire un rayon de lumière dans un nid de hiboux ; il ne sert qu'à blesser leurs yeux et à exciter leurs cris $»^{12}$.

Dès lors, l'enjeu commun que semble constituer, pour les philosophes $\mathrm{du} \mathrm{XVIII}^{\mathrm{e}}$ siècle, l'expansion sociale de la raison, est en fait doublement problématique : premièrement, parce que bien qu'ils aient en vue l'utilité du genre humain, les progrès partiels des connaissances ne dissipent pas nécessairement la double inégalité sociale et épistémique des conditions ; deuxièmement, parce qu'en repoussant la superstition, on risque ou bien de

7. « (...) je n'ai guère que du bien à dire du temps présent, en dépit de tant de gens d'esprit qui ne vantent que le passé ", avoue la Vérité à sa mère la Raison (Voltaire, Éloge historique de la raison, in $O C V$., éd. cit., vol. 76, 2013, p. 376-377).

8. Rousseau, Discours sur les sciences et les arts, in Euvres complètes, Paris, Gallimard, 1959-1995, t. III, p. 7.

9. Voltaire, Homélies prononcées à Londres, «Première homélie », in $O C V$, éd. cit., vol. 62, p. 437.

10. Op. cit., p. 427.

11. C'est ce que ne manquera pas de souligner, en réponse à Voltaire, la Correspondance littéraire de juin 1767: «L'Église métropolitaine et primatiale des athées de Paris a crié au scandale à propos de la première homélie. Elle a prétendu que le patriarche, avec son rémunérateur et vengeur, n'était qu'un capucin, et que c'était poser les fondements de la morale sur une base bien fragile que de l'établir sur de tels principes ».

12. Diderot, La Promenade du sceptique, in Euvres, éd. cit., vol. 1, p. 74. 
se heurter à la résistance de celle-ci (et on verra à nouveau s'accentuer la distinction des simples et des esprits forts), ou bien de voir la raison se transformer en une simple puissance de dissolution de la collectivité.

\section{La «fureur de parler et d'écrire": Deschamps critique des philosophes}

Pour développer ces deux difficultés, on en appellera alors à une œuvre susceptible de jouer ici le rôle d'un révélateur: celle de Léger-Marie Deschamps, qui travaille dans l'obscurité du siècle tout en se confrontant pied à pied avec ses têtes philosophiques. Rappelons brièvement les thèses du « gros bénédictin » cher à Diderot, qui voyait en lui un «moine athée » et un "apôtre du matérialisme $»^{13}$. D'une part, il élabore un système métaphysique qui entend formuler un athéisme éclairé: Dieu est alors assimilé à l'existence négative (le Rien, ou encore Tout comme négation de toute relation à des parties qui le composeraient), elle-même condition de possibilité de l'existence des êtres dans leur totalité (le Tout, c'est-à-dire la sommation de toutes les existences particulières) comme dans leur particularité (les êtres singuliers qui composent ce tout). D'autre part, il en déduit une structure historique ternaire. À l'état sauvage succède d'abord l'état de lois, marqué par une " inégalité morale, ou sociale, qui le constitue et qui, poussée à un point excessif, en fait depuis des milliers d'années l'état social le plus abominable possible ${ }^{14}$; il faut alors en venir à l' «état de mœurs », caractérisé par l'absence de lois et d'autorités, l'égalité morale et la communauté des biens. Ces deux versants de la pensée de Deschamps sont, on y reviendra, articulés l'un à l'autre: la vérité métaphysique est la condition de possibilité de la transformation sociale et du passage des lois aux mœurs. La manifestation de cette vérité doit alors rendre possible l'égalisation des conditions ; et inversement, il ne saurait y avoir de lumières également réparties dans le corps social sans que ce dernier soit lui-même défini par la stricte égalité - non seulement épistémique ou juridique, mais encore sociale et politique - de ses membres.

Comment, d'une part, faire que le progrès du savoir soit en même temps l'émancipation des individus, et non pas l'accentuation des distinctions? Et, d'autre part, quel type de société est possible dès lors que la diffusion des connaissances signifie potentiellement en même temps, sous la forme de la critique du préjugé, la dissolution des opinions communes? La réponse qu'élabore Deschamps constitue alors une tentative de synthèse : la vérité est égale pour tous, et tous sont égaux pour la vérité. Mais cette position, dans laquelle il est tentant de reconnaître l'affirmation radicale et substantielle

13. Op. cit., vol. 5, p. 969.

14. Léger-Marie Deschamps, Euvres philosophiques, Paris, Vrin, 2 vol., 1993, p. 110 (désormais abrégé $O P$ ). 
de l'idéal des Lumières, implique en fait une critique de la philosophie en tant que celle-ci prétend se distinguer du vulgaire : la vérité «manifestée aux hommes, serait moins un sujet de triomphe pour les esprits forts contre les croyants que pour les croyants contre les esprits forts, quoiqu'elle soit destructive de toute loi morale $»^{15}$. Comment comprendre une telle affirmation? Et quel type d'égalité suppose-t-elle pour les individus auxquels la vérité est supposément appelée à se manifester?

Dès l'abord, on l'a vu, le système de Dom Deschamps se présente comme une critique de la structure sociale en tant que celle-ci est un « état de lois ", c'est-à-dire de division et de subordination des hommes les uns aux autres. Il est donc vain d'en appeler à la diffusion des lumières de la philosophie, étant donnée l'inégalité effective de ceux parmi lesquels ces dernières doivent circuler. D'accord en cela avec le Rousseau du Discours sur les sciences et les arts (1750), Deschamps note que « presque toutes nos connaissances sont de trop aux yeux de la saine raison, qui les considère non pas relativement aux besoins excessifs que nous avons aujourd'hui, mais relativement aux besoins modiques que nous aurions, si la vérité était à la place de l'absurde, si nous avions des mœurs au lieu de lois ${ }^{16}$. Sans doute les sciences et les arts doivent-ils être rapportés aux besoins humains auxquels ils viennent répondre : ainsi en usent d'ailleurs les Philosophes qui, nommant ce rapport «utilité », entendent précisément justifier que le développement et la circulation du savoir correspondent à l'amélioration du corps social dans son ensemble. Mais Deschamps renverse le rapport: les connaissances humaines ne sont pas tant ce qui permet la satisfaction des besoins que l'instance de leur production. Et c'est pourquoi l'extension de ces connaissances est finalement problématique : elle correspond non pas à la dissipation des ténèbres mais à une multiplication de sollicitations artificielles auxquelles l'individu ne peut simultanément se consacrer. Le raffinement et la complexification des savoirs fonctionnent alors selon un modèle à la fois dispersif (puisqu'on ne peut répondre à la fois à tous les désirs qu'engagent nos vaines connaissances) et privatif (en devenant plus abstraites et plus complexes, les connaissances passent hors de la portée du commun). Loin de conclure à l'insuffisance cognitive du vulgaire, c'est au contraire la vanité des progrès multiples et sectoriels des sciences et des arts que souligne Deschamps : «il est constant que nos connaissances sont toujours d'autant plus vaines qu'elles sortent plus de la sphère de celles du peuple, que leurs objets sont plus hors de nous, qu'ils sont moins sous nos yeux, sous nos mains, sous nos pas, qu'ils sont plus dans le lointain; d'où il s'ensuit que c'est une folie à nous de les avoir poussées au point que nous l'avons fait, et de chercher toujours à les pousser au-delà, comme nous le faisons $\gg{ }^{17}$. Contre la spécialisation des savoirs, il faut donc envisager l'égalisation, aussi bien épistémique que sociale, des conditions : l'écart du

15. Op. cit., p. 87.

16. Op. cit., p. 290 , note e.

17. Op. cit., p. 291. 
savant et du vulgaire est le signe non d'un déficit du second, mais d'un excès du premier.

Dès lors, le rôle social revendiqué par les Philosophes ne peut finalement être compris que comme une tentative de singularisation et de distinction. C'est ce qu'affirment avec netteté les Lettres sur l'esprit du siècle (1769): les Philosophes, "par les lumières qu'ils ont au-dessus du vulgaire », prétendent dissiper la superstition, mais ils sont en fait en proie à « cette fureur de parler et d'écrire qui n'a foncièrement sa raison que dans celle de ne pas laisser ignorer qu'ils sortent de la classe des autres hommes, et qu'ils ne sont pas des gens à qui on en fait accroire, comme à leurs Valets, leurs Cordonniers et leurs Tailleurs $\gg{ }^{18}$. Le savoir des beaux esprits redouble alors, dans l'espace intellectuel, la division hiérarchique du maître et du serviteur: si l'un veut bien éclairer l'autre, c'est à la condition expresse de ne pas être confondu avec lui, de sorte qu'en prétendant égaliser les intelligences, on signale en fait sa propre supériorité.

Il s'agit finalement, en montrant leur insuffisance, de transformer les « demi-lumières » en vérité pleine et entière. Une telle démonstration peut se faire aussi bien d'un point de vue extérieur à celui des Philosophes (ainsi des Lettres sur l'esprit du siècle, qui annoncent prendre le parti de la religion chrétienne contre ceux qui l'attaquent) que selon leur logique propre (ainsi des textes que Deschamps donne à lire à ses interlocuteurs et à ses disciples, qui doivent composer ensemble l'exposé total de la doctrine, recueillie dans un livre intitulé sobrement le Vrai système). Dans les deux cas, l'enjeu est le même : mener à son terme ce qui n'a été accompli qu'à moitié - cette première moitié étant du reste, par son incomplétude même, la plus risquée :

Une philosophie telle que celle qui règne peut bien occasionner une révolution dans la religion, dans les mœurs et dans le gouvernement, mais elle ne peut rien de plus avec ses demi-lumières : car cette révolution, toujours à éviter comme aussi dangereuse qu'inutile, n'empêcherait pas l'état de lois divines et humaines de subsister, et le mal moral, dont cet état est la cause, d'exister avec la même force, quoique sous d'autres formes ${ }^{19}$.

Autrement dit, c'est non seulement dans son contenu théorique, mais encore dans son effectivité historique et sociale que le projet philosophique des hommes des Lumières doit être critiqué. Faute de pouvoir mettre à bas "l'état de loi divines et humaines ", le but qu'il se donne se trouve inaccessible, et les moyens qu'il emploie sont non seulement inopérants,

18. Dom Deschamps, Lettres sur l'esprit du siècle, II ${ }^{\text {ème }}$ lettre, Londres, 1769, p. 35 (désormais abrégé $L E S$ ). La critique n'est pas sans rappeler l'apostrophe de Rousseau dans le Discours sur les sciences et les arts («Ô, fureur de se distinguer, que ne pouvez-vous point?»). Dans un cas comme dans l'autre, la dénonciation de l'orgueil comme mobile du savoir ne va pas, bien sûr, sans résonances chrétiennes (cf. sur ce point l'article de B. Binoche, "Rousseau : le premier Discours sans le second», in Revue de Théologie et de Philosophie, vol. 151, n 4, févr. 2020, p. 319-336).

19. Dom Deschamps, $O P$, p. 83. 
mais encore périlleux. Comment comprendre que la «révolution» que cherchent pourtant à produire les Philosophes, loin de constituer une rupture décisive, ne soit rien d'autre qu'une agitation purement circulaire, que la reconduction, sous d'autres formes, du même état social que l'on se proposait pourtant d'améliorer?

\section{Théisme, athéisme, domdeschampisme}

C'est en fait d'abord une inconséquence de principe qui est selon Deschamps en cause - et c'est à ce point qu'on retrouve, après la question de l'inégale progression des savoirs, celle de l'usage social de la croyance. Sans doute le philosophe ne ménage-t-il pas ses efforts pour délivrer le peuple crédule de l'emprise de la superstition et du pouvoir des prêtres: débarrassée du fatras de préjugés irrationnels dont le christianisme et ses mystères l'ont recouverte, l'idée d'une divinité juste ne peut-elle pas alors prétendre devenir le socle d'une société éclairée ? Or en substituant ainsi à la religion révélée une religion supposément naturelle, on laisse intact le fondement effectif dont toute religion tire sa puissance de domination, et qui n'est autre que l'institution des lois :

Il nous a fallu nécessairement les lois positives pour avoir la notion du juste et de l'injuste, du bien et du mal moraux, et c'est uniquement par cette notion que nous avons pu avoir celle d'un Dieu rémunérateur et vengeur. (...) Si ces lois viennent des hommes, comme elles en viennent en effet, la première de ces deux notions a précédé la seconde, venue depuis à son soutien; et elle a été l'effet de la loi d'un homme faite à un autre homme : car cette loi d'où il résulterait qu'on ferait bien en l'observant, et mal en ne l'observant pas, donnait nécessairement par elle-même la notion du bien et du mal, du juste et de l'injuste ${ }^{20}$.

La genèse de l'unité sociale produite par la religion est en dernière instance elle-même sociale : la loi, sous son double aspect humain et divin, positif et religieux, est toujours "celle d'un homme faite à un autre homme ». Ainsi, la religion supposément naturelle, loin d'exprimer la vérité que la religion révélée recouvre sous un tissu d'absurdités, reconduit alors plutôt l'arbitraire du commandement en général. On pourra donc aller jusqu'à identifier, sur le plan théologique, la loi et le péché lui-même : cette dernière est déjà la transgression qu'elle pose $\mathrm{e}^{21}$.

20. Op. cit., p. 187.

21. « «Il faudrait (...) qu'elle [la théologie] établît que la loi est le péché, comme elle l'est en effet; et c'est ce qu'elle ne pourrait pas établir sans faire manifestement contre elle-même "», op. cit., p. 190. 
Est-ce à dire que l'on devra diffuser l'athéisme ? Nullement : celui-ci « n'a de commun avec la Vérité que de rejeter toute religion », et dès lors, « il détruit tout, sans rien établir $»^{22}$. Sans doute faut-il bien souscrire, avec Spinoza et les athées, à la critique de l'idée d'une divinité anthropomorphe, qui d'une "notion mal développée » (celle de l'existence totale des êtres) fait « un être intelligent et moral à son image, un Dieu architecte, rémunérateur et vengeur, après l'avoir fait législateur $»^{23}$. Mais il faut en même temps critiquer un tel type de savoir, purement négatif: loin de constituer la mise en commun du vrai, il s'agit plutôt d'un renoncement à la vérité elle-même. Et c'est pourquoi l'homme éclairé est encore un homme de préjugés :

L'athéisme (...) nie ce que nous appelons Dieu, et c'est par là qu'il est audessus de tous les préjugés de la religion, mais il lui reste le préjugé qu'il ne reste rien de Dieu, de ce point de vue anéanti : c'est-à-dire que la vérité n'existe point comme principe universel, et ce préjugé sera peut-être plus fort dans lui contre la vérité que tous les préjugés de religions dans la tête des croyants; c'est à l'évidence à vaincre tous ces obstacles que lui oppose notre ignorance ${ }^{24}$.

L'athéisme apparaît finalement comme une «philosophie destructive, sans connaissance de cause ${ }^{25}$, incapable de substituer un principe véritablement systématique à l'idée de divinité qu'il conteste. Sans doute, théoriquement, l'idée d'une divinité souveraine sert-elle de principe à l'édifice social dans son ensemble, dans la mesure où elle sanctionne le système des lois, des châtiments et des peines, des distinctions et des récompenses - bref, cet état d'inégalité et de " désunion dans l'union » qui se nomme "état de lois ». Mais pratiquement, la genèse est, on l'a vu, inverse: c'est l'état de division sociale, et les lois que se donnent les hommes les uns aux autres, qui fournissent finalement le contenu théorique de l'idée d'un Dieu souverain.

Autrement dit, ce qui valait contre le théiste - le cercle vicieux des lois positives et de l'idée d'un Dieu rémunérateur et vengeur, où la domination produit elle-même son propre fondement théorique - vaut a fortiori contre l'athée: si le premier prend le cercle vicieux pour un cercle vertueux, le second ne voit pas de cercle du tout et pense pouvoir se contenter de détruire l'idée de divinité en maintenant l'autre pôle avec lequel elle fonctionne, celui des lois. Or pour dénouer un tel enchevêtrement, un double mouvement serait nécessaire. D'une part, il faut comprendre que contester théoriquement le Dieu souverain, c'est en même temps contester pratiquement l'édifice social de «l'état de lois »- et Deschamps se lamente : si l'athéisme « avait au moins eu la vérité morale, au défaut de la vérité métaphysique ; s'il avait eu l'état de mœurs, l'état d'égalité, ou de loi naturelle morale, à mettre à

22. Op. cit., p. 88.

23. Op. cit., p. 112.

24. Op. cit., p. 88.

25. Op. cit., p. 83. 
la place de l'état de lois humaines, (...) il eut été fort contre le théisme $»^{26}$. Mais, d'autre part, découvrir cet "état de mœurs » implique en même temps d'en penser le principe théorique - c'est-à-dire d'en venir à la vérité métaphysique que Deschamps prétend énoncer; dans ces conditions, bien qu'on puisse déplorer l'inconséquence de l'athée qui croit pouvoir « détruire les lois divines en conservant les lois humaines qui leur ont donné leur être $»^{27}$, elle n'est en dernière instance pas surprenante. C'est qu'il faut comprendre, notamment contre l'auteur du Système de la nature (1770), que l'athéisme est nécessairement métaphysique, de sorte qu'on peut dire des athées qu'ils sont «comme le Bourgeois gentilhomme, qui parlait prose sans le savoir»: "vous êtes métaphysiciens, comme athées, et vous l'ignorez au point que vous niez l'existence de la métaphysique », s'exclame Deschamps $^{28}$ ! C'est alors seulement à la condition expresse d'assumer son propre contenu métaphysique que quelque chose comme un "athéisme éclairé $"{ }^{29}$ est possible : ainsi, on ne se contentera pas (négativement) de nier l'existence divine, mais on affirmera plutôt que « Dieu est le Rien, le néant même $»^{30}$ - c'est-à-dire l'existence négative qui est la condition de possibilité, le «fin fonds », de tout ce qui est. Ainsi, la doctrine ne peut se présenter que comme un système, que les interlocuteurs de Deschamps au château des Ormes nommeront plaisamment le « domdeschampisme » ou le « riénisme » : elle doit fonder en principe l'égalité morale des individus dans l'état de mœurs sur l'égalité ontologique des parties du Tout.

\section{La vérité toute nue}

On aura alors définitivement compris que la «fureur» qu'ont les philosophes de se distinguer du vulgaire correspond à une impossibilité de ne pas jouer le jeu des distinctions sociales, qui est celui de l'état de lois. Si l'apôtre de l'état de mœurs cherche à tendre à l'égalité par l'égalité ${ }^{31}$, les philosophes, pour leur part, risquent de tendre à l'égalité par l'inégalité : on retrouve ici la figure de l'ambitieux, qui certes «n'a pas pour premier objet d'avoir de la supériorité sur ses semblables, mais de n'avoir aucun de ses semblables qui ait de la supériorité et de l'avantage sur lui ${ }^{32}$. Or une telle disposition affective reproduit à son tour la tutelle qu'elle cherche à secouer, car « il est dans la nature de notre état de lois de ne pas pouvoir être sans

26. Op. cit., p. 88.

27. Op. cit., p. 113.

28. Op. cit., p. 222.

29. Op. cit., p. 241.

30. Op. cit., p. 235.

31. Il en va ainsi du «petit nombre d'homme qui ont les vertus morales, qui ne rapportent à eux qu'en rapportant aux autres, qui aiment leurs semblables, qui tendent à l'égalité par l'égalité, et non par l'inégalité, comme le font les ambitieux» (op. cit., p. 248 ; nous soulignons).

32. Op. cit., p. 248 , note n. 
maître qu'on ne soit le maître $\rangle^{33}$. La revendication du droit de chacun à penser par et pour lui-même doit alors être éclairée sous un jour critique : la diffusion parmi le peuple des lumières philosophiques, si elle n'est pas menée jusqu'au terme que lui assigne Deschamps, est suspecte de reconduire l'ordre social - la distinction du juste et de l'injuste, du tien et du mien, et partant la domination de l'homme sur son prochain. Aussi,

(...) si l'esprit qui règne aujourd'hui porte les hommes à être indépendants, il les porte en même temps à être personnels et attachés à leurs propriétés. De là, nulle matière à crainte pour les puissances que cet esprit ne fasse corps, et toute voie ouverte à sa force pour le châtier et l'anéantir même, quand elle voudra user de sa force. Cet esprit crie au despotisme, sans songer que lui-même peut l'occasionner, en mettant la puissance dans le cas de connaître tout ce qu'elle peut ${ }^{34}$.

La critique philosophique apparait alors doublement inoffensive : d'une part, l'indépendance qu'elle promet à la raison est une indépendance personnelle, qui maintient finalement la structure divisée et atomistique de l'état de lois. Elle suppose, au moins implicitement, une épistémologie de propriétaire - chacun trouve en sa raison son bien propre -, qui doit selon Deschamps être combattue par une métaphysique du commun. Mais même à supposer qu'elle entraîne les individus à questionner l'ordre établi en affirmant leur indépendance, elle les rend d'autre part vulnérables à la coercition des « puissances ». Elle apprend au maître à se méfier de celui qui le sert, lui révèle les voix de la contestation, et « faute que cet esprit ne fasse corps » (son efficace étant strictement individuelle, c'est-à-dire dispersive, l'incarnation d'une puissance commune en face des puissances constituées s'avère donc impossible), elle offre des victimes à son courroux. C'est qu'il faut comprendre que « l'état de lois n'a point été un état de convention, c'est la force qui l'a formé, c'est la force qui l'entretient $\gg{ }^{35}$. Aussi est-ce bien en vain qu'on essaiera d'en appeler aux lumières de chacun contre cette force, dans la mesure où celle-ci demeurera libre d'écraser celles-là si elles se trouvent en travers de sa route : la raison du plus fort est toujours la meilleure.

Se laisse voir ici le double rapport au savoir qu'entretiennent d'une part les puissants, d'autre part ceux qui leur sont soumis. En ce qui les concerne, les simples sujets s'exposent en avançant trop à découvert : revendiquer pour chacun la liberté de penser et de parler, sans ôter aux puissants la liberté de contraindre, c'est être inconséquent, hypocrite ou fou ${ }^{36}$. C'est pourquoi la seconde des Lettres sur l'esprit du siècle s'érigera contre la liberté de

\section{Ibid.}

34. Op. cit., p. 247, note 1.

35. Op. cit., p. 286.

36. «La demande de vivre dans l'indépendance des lois mènerait tout droit aux PetitesMaisons », LES, p. 33. 
penser - au scandale de Diderot, qui ne renoncera vraisemblablement au compte-rendu assassin qu'il avait prévu de donner de l'ouvrage dans la Correspondance littéraire qu'en apprenant qui en était l'auteur ${ }^{37}$. Ainsi, affirme Deschamps, cette liberté que demandent les philosophes, «les lois civiles ne peuvent leur accorder sans aller contre leur essence $»^{38}$ : l'argument est apparemment présenté à l'appui de la religion et du gouvernement, mais peut bien sûr être inversement compris comme un défi à aller jusqu'au bout du raisonnement, en s'en prenant à cette essence des lois elle-même. Aussi a-t-on intérêt à déguiser sa critique si l'on n'est pas prêt à la mener jusqu'au bout. Mais, symétriquement, le pouvoir a lui aussi intérêt à ne pas dire tout ce qu'il sait :

La division des sujets du prince en différents états est le grand point d'appui de sa domination, mais le point d'appui, tout grand qu'il est, ne lui suffit pas, il faut nécessairement qu'il ait toujours tels et tels de ses sujets à diviser entre eux, pour le maintien de sa puissance, et cette division sourde, qui est le secret du ministère, est ignorée des peuples et presque toujours même des grands et des gens en place que l'on divise ${ }^{39}$.

La domination doit alors masquer, jusque dans ses propres rangs, le principe de division sur lequel elle repose: son origine est matérielle (l'inégalité du fort et du faible), mais sa préservation dépend bien d'un ressort cognitif - le secret quant à son propre fonctionnement.

C'est pourquoi, même mise au jour une fois de plus l'impuissance de la «philosophie destructive », on continuera finalement à en appeler à la manifestation du vrai comme catalyseur de la transformation sociale. Mais ce n'est plus ni l'accumulation inutile des connaissances (c'est-à-dire les progrès des sciences et des arts) ni la critique de la superstition et du préjugé (c'est-à-dire la défense de la liberté de penser contre la tutelle théologique) qui sont alors convoquées, mais la propagation de la « vérité simple et nue ». Le passage de l'absurde au vrai (et, parallèlement, de l'état de lois à l'état de mœurs) est conçu comme une discontinuité entre deux pôles contradictoires, de sorte qu'au modèle pluriel de la diffusion des connaissances se substitue celui de la manifestation du vrai dans son unicité :

La vérité, par un effet de l'absurde qui règne et qu'elle a à combattre, est un protée : on ne la saisira qu'après qu'elle se sera montrée sous plusieurs formes, mais une fois saisie on jugera que c'est l'évidence même.

C'est par elle qu'on aura le mot qui, une fois deviné, se trouvera être le mot de tout ce qui a été, jusqu'à présent, énigme pour les hommes $(\ldots)^{40}$.

37. Voir la lettre que Diderot avait prévu d'envoyer au lieutenant de police Sartine, avant de se raviser (in L.-M. Deschamps, Correspondance générale, Paris, Honoré Champion, 2006, p. 268-269).

38. LES, p. 33.

39. OP, p. 267.

40. Op. cit., p. 85. 
Il y a alors bien une temporalité du vrai : prospectivement multiple et protéiforme (c'est-à-dire avant que le Vrai système en a donné le mot), il est rétrospectivement simple et autosuffisant (c'est-à-dire une fois que sa manifestation a eu lieu). Mais cette temporalité est en même temps une temporalité sociale :

Nous sommes dans un siècle de demi-lumières, où nous avons un besoin particulier que notre ignorance soit vaincue. La révolution que pourrait occasionner la vérité donnée aux hommes parerait à celle dont nous sommes menacés, et ce serait alors un très grand bien qui parerait à un grand mal. Cette heureuse révolution ne peut pas arriver tout d'un coup : mais la vérité, manifestée et avouée de proche en proche, y porterait les esprits et les détournerait d'en désirer une autre ${ }^{41}$.

Sans doute la manifestation n'a-t-elle pas lieu de façon instantanée ; toutefois, quelle que soit la durée que la vérité demande pour se propager, cette propagation elle-même n'en est pas moins irrésistible, car la doctrine qu'élabore Deschamps a pour elle «le despotisme de son évidence ${ }^{42}$. Sans doute Deschamps semble-t-il s'exprimer ici avec une conviction naïve : en fait de simplicité, l'articulation métaphysique des parties, du Tout et de Tout ne laissera pas de comporter quelques obscurités, qui rebuteront nombre des interlocuteurs auxquels le moine s'adressera. Mais face aux reproches qui font du Vrai système une métaphysique absconse, Deschamps va jusqu'à un renversement spectaculaire : c'est justement à cause de sa simplicité que la doctrine est difficile à comprendre ${ }^{43}$ ! On se gardera toutefois bien de voir là l'expression d'un orgueil personnel : le ton impérieux (que déplorera le marquis de Voyer d'Argenson ${ }^{44}$ lorsqu'il tentera de perpétuer la doctrine de son protégé après la mort de celui-ci) est un effet du système lui-même.

Car c'est finalement la double extériorité - logique et ontologique - du vrai qui est ici en cause : la vérité n'est pas un produit partiel et contextuel de la raison, mais l'ordre même des choses, indépendamment de l'existence humaine qui le conçoit. C'est pourquoi les «Réflexions métaphysiques préliminaires » peuvent affirmer : " c'est l'homme qui développe ; c'est le fond qu'il a de commun avec tous les êtres qui est développé ${ }^{45}$. La vérité est indépendante de celui qui l'énonce: sa difficulté apparente recouvre

41. Op. cit., p. 243.

42. Op. cit., p. 130, note $\mathrm{m}$.

43. Voir la lettre au marquis de Voyer d'Argenson du 26 avril 1765 : « [m]a philosophie est trop neuve, trop simple, trop opposée à tout système et trop facile à comprendre pour être facilement comprise », in L.-M. Deschamps, Correspondance générale, éd. cit., p. 110.

44. Marc-René de Voyer d'Argenson (1722-1782), en qui Deschamps trouva sinon un adepte, du moins un protecteur toujours disposé à l'accueillir au château des Ormes. Il s'agit du fils du comte d'Argenson (1696-1764), ministre de la Guerre de Louis XV destitué en 1757 par la Pompadour après l'attentat de Damiens, et dédicataire de l'Encyclopédie en tant que directeur de la librairie.

45. Op. cit., p. 96, note b. 
en fait sa simplicité, de sorte qu'elle peut et doit être considérée comme commune à tous. Ainsi, le passage à la métaphysique, s'il va à rebours de l'empirisme largement défendu par ceux des contemporains de Deschamps qui se revendiquent de la philosophie, permet supposément d'éviter le double écueil qui a été opposé à celle-ci : son caractère distingué, c'est-àdire socialement situé, et son incapacité à pousser jusqu'au bout la critique sociale de la distinction des conditions.

En ramenant la vérité à sa simplicité métaphysique, on en permettrait alors l'usage commun :

La vérité métaphysique et éternelle, ou, si l'on veut, une et unique, que je développe, est tellement vérité qu'il n'y a rien à lui opposer. Elle serait d'un développement si court et si facile pour des âmes nettes de préjugé, que rien ne serait plus aisé aux pères que de la transmettre à leurs enfants, dans l'état de mœurs, où elle seule peut amener, aujourd'hui que l'absurde nous tient sous l'état de lois. Mais qu'il en coutera, peut-être, pour se persuader que quelque chose d'aussi simple, d'aussi grammatical, soit la vérité tant désirée et tant cherchée $!^{46}$

Ainsi, l'état de mœurs est un état marqué non seulement par la possibilité, mais encore par l'effectivité d'un savoir partagé - et c'est précisément ce savoir qui doit abolir l'inégalité des conditions en même temps que le pouvoir qu'ont certains d'en dominer d'autres. La transmission entre les générations en est simplement orale : l'instruction, réduite à sa portion congrue, devient naturelle et facile. Mais aussi bien, cette simplicité terminale du savoir fonctionne également comme l'épuration de ce qui, en lui, n'est pas absolument nécessaire - c'est-à-dire de tout ce qui n'est pas le Vrai système! Il conviendra alors de sacrifier non seulement la superstition théologique, mais encore les sciences et les arts eux-mêmes, sur l'autel de l'égalité morale - car c'est seulement ainsi, note Deschamps, qu'on pourra parvenir à l'état de mœurs désiré :

Il faudrait, pour y entrer, brûler non seulement nos livres, nos titres et papiers quelconques, mais détruire tout ce que nous appelons les belles productions de l'art. Le sacrifice serait grand, sans doute, mais il faudrait le faire : car à quoi bon laisser subsister des monuments qui ne seraient plus d'aucun usage et qui, en prouvant notre intelligence à nos descendants, leur prouveraient notre folie, et nuiraient même à l'objet, utile pour eux, de les éloigner de toute idée de nos mœurs? Je choque par là les idées de la partie des hommes prétendue la plus excellente, de ce qu'on appelle les hommes cultivés, de cette petite partie qui, très distinguée du peuple qu'elle domine et dont elle tire sa subsistance, ses commodités et son luxe, se croit une raison bien supérieure à celle du peuple... ${ }^{47}$

46. Op. cit., p. 239 , note o.

47. Op. cit., p. 299. 
À nouveau, l'hypothèse de la suppression des produits de l'esprit humain, et donc des livres eux-mêmes en tant qu'ils sont doublement superflus - eu égard à la double simplicité des besoins humains et de la vérité métaphysique - fait signe vers l'inégalité sociale qui sous-tend la distinction des hommes cultivés et du peuple. Puisqu'elle suppose que ce dernier fournit aux autres leur subsistance, la production des savoirs apparaît en dernière instance comme émanant d'une relation d'exploitation; arts et sciences peuvent finalement être versés au compte des dépenses somptuaires du genre humain, dont la frugalité de l'état de mœurs devra bien sûr se passer. Dans l'égalité absolue du monde sans lois imaginé par Deschamps, l'édifice des connaissances humaines produites par l'inégalité perd alors sa valeur intrinsèque, à l'exception de celles qui sont directement utiles à la satisfaction des besoins : ainsi le communisme agraire de l'état de mours admet-il encore des forges, mais il se passe de la physique ${ }^{48}$, de l'histoire ${ }^{49}$ et même des calendriers ${ }^{50}$. Pour que les hommes soient égaux entre eux, y compris dans leur partage d'un savoir commun, il faut en dernière instance prendre au pied de la lettre l'expression de "vérité nue », et se dépouiller des « arts agréables » dont s'orne l'état de lois :

On a toujours dit que la vérité était faite pour paraitre toute nue : cela s'étend beaucoup plus loin qu'on ne l'a pensé, puisqu'il est vrai qu'elle rejette non seulement toute parure dans le discours, mais toute harmonie factice qui sort de l'ordinaire ${ }^{51}$.

On retiendra alors de Dom Deschamps une leçon paradoxale. D'une part se déploie dans son œuvre une critique de l'efficace sociale du travail de la Philosophie : ses lumières ne suffisent pas, à elles seules, à émanciper les hommes qu'on se propose d'instruire; encore faut-il qu'elles soient rapportées aux conditions dans lesquelles elles sont produites pour que leur programme de transformation sociale ne demeure pas lettre morte. De ce point de vue, Deschamps propose finalement un passage à la limite à partir des prémisses communes de ses contemporains : si elle veut vraiment avoir en vue l'intérêt des hommes, l'expansion de la raison ne saurait s'arrêter à une critique de la religion, et doit en venir à une archi-critique de l'ordre social lui-même. Mais en retour, Deschamps se heurte aux limites

48. « (...) qu'auraient-ils besoin de la science des Copernic, des Newton, et des Cassini ?» (op. cit., p. 319).

49. «Les siècles passés seraient à leur égard comme s'ils n'avaient pas été [...]» (op. cit., p. 311).

50. « (...) ils vivraient sans se soucier de compter les jours et les années ; ce qui les mettrait dans le cas d'ignorer l'époque de leur naissance, et de moins prévoir le dernier moment, où, sans infirmité, ils passeraient de la vie à la mort, comme on passe de la veille au sommeil » (op. cit., p. 319).

51. Op. cit., p. 310. On retrouve une formulation analogue à la suite d'un passage cité plus haut: «On a toujours dit qu'elle [la vérité] était faite pour paraître toute nue, et que tout ornement lui était étranger : je laisse à juger si je n'ai pas prouvé qu'on avait eu raison de le dire » (op. cit., p. 239). 
de ses propres présupposés : la manifestation de la vérité métaphysique est tenue pour être, en elle-même, capable d'opérer la transformation qu'elle énonce, sans que les conditions matérielles de sa propre effectuation soient alors elles-mêmes réfléchies. Sans doute un tableau au moins sommaire du genre de société que sera l'état de mœurs est-il possible ; mais les moyens politiques d'y parvenir sont suspendus à la seule formulation des principes théoriques qui la fondent, c'est-à-dire en dernière instance à l'écriture et à la diffusion d'un seul livre, le Vrai système. Aussi, si du côté de l'état de lois, c'est l'ordre social qui détermine la production et l'économie du savoir, du côté de l'état de mœurs c'est au contraire la vérité qui, par sa force propre, doit renouveler tout l'édifice : la critique que Deschamps fait porter sur la philosophie peut ainsi lui être retournée. À supposer que le Vrai système la contienne réellement, est-il suffisant de tirer la vérité du fonds du puits pour la montrer aux hommes, comme le prétend le titre d'un des ouvrages de Deschamps ? On se gardera sans doute de penser qu'une telle exhumation puisse être quelque chose comme un basculement historique irréversible et définitif - et on se rappellera que la raison, qui comme la vérité sa fille aime à séjourner au fond des puits, se réserve toujours une fois qu'elle en est sortie la possibilité d'y retourner pour peu que « des orages surviennent $»^{52}$. 